\title{
INTERVENCIÓN COGNITIVO CONDUCTUAL PARA PACIENTES ADOLESCENTES Y SUS PADRES EN EL TRATAMIENTO DE LA BULIMIA NERVIOSA
}

\author{
Úrsula Castro, Cristina Larroy y M. Ángeles Gómez
}

Facultad de Psicología de la Universidad Complutense de Madrid

\begin{abstract}
Resumen: Se evalúa la eficacia de un programa de intervención para adolescentes bulímicas compuesto por un tratamiento cognitivo-conductual para las pacientes (12 sesiones) y un grupo psicoeducativo paralelo para sus respectivos padres (12 sesiones). El estudio se llevó a cabo con 21 pacientes adolescentes diagnosticadas de bulimia nerviosa que acudían a la Unidad de Trastornos Alimentario del Hospital del Niño Jesús (Madrid, España) y con sus padres. El grupo control estuvo constituido por 17 pacientes, de similares características, cuyos padres no recibieron intervención. Los resultados demuestran que esta modalidad de intervención es altamente eficaz en la reducción de los síntomas centrales de la bulimia nerviosa y la sintomatología asociada manteniéndose los resultados al año de seguimiento, y que es más eficaz que un grupo control (consistente en tratamiento solo de las pacientes) en cuanto a los abandonos y recaídas, encontrándose diferencias estadísticamente significativas en estas dos variables.
\end{abstract}

Palabras Clave: Bulimia nerviosa, tratamiento, grupo de padres, terapia cognitivo-conductual.

\section{Cognitive behavioral and parental group intervention for adolescents in the treatment of bulimia nervosa}

\begin{abstract}
The objective was to evaluate the effectiveness of an intervention program for adolescents with bulimia composed of a cognitive-behavioral treatment for patients (12 sessions) and a psychoeducational group activity with their parents (12 sessions). The study was conducted with 21 adolescent patients, diagnosed with bulimia nervosa, of the Eating Disorders Unit at the Hospital del Niño Jesus (Madrid, Spain), and their parents. The control group consisted of 17 patients with similar characteristics, whose parents received no intervention. The results show that this form of intervention is highly effective in reducing the core symptoms of bulimia nervosa and associated symptoms, maintaining the result at one year follow up, and that it is more effective than the control group (consisting in treatment for patients only) as regards dropouts and relapses, finding some statistically significant differences in these two variables.
\end{abstract}

Key words: Bulimia nervosa, treatment, parental group, cognitive-behavioral therapy.

\section{INTRODUCCIÓN}

La bulimia nerviosa es un trastorno grave y complejo, en cuya etiología multi-factorial se ven implicados factores biológicos, psicológi-

\footnotetext{
Recibido: 26-agosto-2009; aceptado: 9-febrero-2010.

Correspondencia: Cristina Larroy García. Facultad de Psicología de la Universidad Complutense de Madrid. Campus de Somosaguas s/n 28223 Madrid. Correo-e: clarroy@psi.ucm.

Agradecimientos: Agradecimiento especial a todos los miembros de la Unidad de Trastornos Alimentarios del Hospital de Niño Jesús por haber permitido la realización de esta investigación y en especial al Dr. Gonzalo Morandé Lavín, por su apoyo a este proyecto.
}

cos y sociales y, en cuyo origen destacan: (a) la presencia de un contexto social y/o familiar de riesgo; y (b) una serie de características personales que hacen a la persona más vulnerable a la práctica de uno de los antecedentes más importantes de la bulimia nerviosa: la dieta restrictiva. Esta conducta, unida a una serie de factores precipitantes, favorecerá el desarrollo de la bulimia nerviosa (Shapiro, Berkman, Brownley et.al., 2007).

En la actualidad existe un importante soporte científico que avala el tratamiento cognitivoconductual para la bulimia nerviosa, especialmente el que deriva del trabajo pionero de Fairburn (1981). Sin embargo, los datos indican 
que más del $60 \%$ de las personas con bulimia nerviosa no recibe el tratamiento adecuado (Wilson, 2005).

Otro dato preocupante es la ausencia de investigaciones sobre la población femenina adolescente, ya que la mayor parte del sustento empírico con el que se cuenta se basa en investigaciones llevadas a cabo con poblaciones adultas. Esto resulta alarmante, sobre todo si tenemos en cuenta que la adolescencia constituye un período evolutivo crítico, ya que las primeras conductas de riesgo aparecen al final de la pubertad, instaurándose el trastorno finalmente en la adolescencia.

En los últimos años ha habido un reconocimiento gradual de la importancia de la participación de las familias en el tratamiento. Se considera que estas intervenciones con la familia deben estar orientadas a la educación e implicación en el tratamiento de sus hijas (Okon, Greene y Smith, 2003). Sin embargo, en la literatura revisada, no se han encontrado datos empíricos sobre el trabajo con padres de pacientes adolescentes, a pesar de que se reconocen, en la familia, variables que contribuyen a mantener el trastorno. Los estudios encontrados hacen alusión a programas para familiares de pacientes con anorexia (Eisler, Le Grange y Asen, 2003; Le Grange, 2005; Lock y Le Grange, 2005) y la eficacia demostrada de dichas intervenciones es más bien limitada (Bryant-Waugh, 2006). El trabajo con las familias otorga un papel importante al entorno del sujeto, considerándolo un factor de mantenimiento fundamental, y consiste en hacer participar en el tratamiento a los familiares de la paciente (Wilson y Fairburn, 1998).

Por todo ello, en la actualidad existe una demanda creciente, por parte de la comunidad científica, de la validación de programas adaptados a la población adolescente que incluyan e impliquen de manera activa a la familia (Asociación Americana de Psiquiatría, 2000a; NICE, National Institute for Clinical Excellence, 2004).

El trabajo con las familias, permite: (a) reducir los sentimientos de aislamiento y soledad de la familia (Colahan y Robinson, 2002; Vandereycken, Castro y Vanderlinden, 1991), (b) el aprendizaje de pautas específicas en relación al manejo del problema de sus hijas (Calvo, 2002); (c) el cambio de actitudes familiares que fun- cionan como variables mantenedoras del problema alimentario de las hijas ,y (d) la creación de un ambiente familiar favorable que facilite el cambio de las pacientes (Gómez, Castro, García, Dúo y Yela, 2003).

En tal sentido, las guías prácticas de la Asociación Americana de Psiquiatría (APA, 2000) aseguran que las intervenciones con la familia pueden ser especialmente eficaces en el tratamiento de la bulimia nerviosa. A pesar de ello, solo se ha encontrado un estudio que ha evaluado su eficacia (Russell, Szmukler, Dare y Eisler, 1987). Los resultados de este estudio dejan mucho que desear, debido a su tasa de abandonos (44\%), tasa explicable, quizá porque el estudio fue realizado con una población adulta, lo que dificulta aún más contar con la familia de la persona para el tratamiento.

Algunos trabajos epidemiológicos otorgan a la familia cierto grado de responsabilidad en el origen y mantenimiento del trastorno (Fairburn, 1995; Iglesias y Romero, 2009; Toro, 1996; Vanderlinden y Vandereycken, 1999; Wilson y Fairburn, 1998;). A pesar de ello, no existen propuestas sistemáticas sobre el trabajo con las familias en la bulimia nerviosa. Fairburn y Cooper (1987) señalan que una de las limitaciones más importantes en el trabajo con familias se debe al hecho de que, en la mayoría de los países europeos, las adolescentes y/o jóvenes dejan el seno familiar a partir de los 20 años aproximadamente. Sin embargo, no sucede lo mismo en España, en donde la mayoría de las jóvenes abandonan el hogar familiar a edades más avanzadas. Esto ha propiciado que la mayoría de los programas de la sanidad pública trabajen de manera paralela con las familias, concretamente con los padres, brindándoles información sobre la enfermedad y pautas específicas a adoptar en relación al problema alimentario de sus hijas. Sin embargo, como ya se ha mencionado, estas intervenciones no forman parte de programas sistemáticamente estructurados.

Con el objeto de paliar esta carencia, se diseñó el presente estudio, cuyo objetivo es la evaluación de un protocolo de tratamiento específico para adolescentes con bulimia nerviosa, que se compone de un tratamiento cognitivoconductual para las pacientes y un grupo paralelo psicoeducativo para sus respectivos padres. 


\section{MÉTODO}

\section{Participantes}

La muestra total estuvo compuesta por 38 mujeres adolescentes diagnosticadas de bulimia nerviosa según los criterios diagnósticos del DSM-IV-TR (APA, 2000b) que asistían a la Unidad Ambulatoria de Trastornos Alimentarios del Hospital del Niño Jesús de Madrid. De ellas, 21 adolescentes componían el grupo experimental: recibieron, además del tratamiento para las pacientes, una intervención paralela con sus respectivos padres $\left(N_{T C C+G P}=21\right)$; y 17 adolescentes constituyeron el grupo control: ellas recibieron el tratamiento cognitivo conductual, pero sus padres no recibieron intervención psicoeductiva $\left(N_{T C C}=17\right)$. El rango de edad oscilaba entre 14-18 años $(M=16,27 ; S D=$ $1,24)$ y la mayoría cumplía criterios diagnósticos de bulimia nerviosa de tipo purgativa $(n=$ 34), siendo el vómito la principal medida compensatoria. El resto $(n=4)$, utilizaba el ayuno y/o el ejercicio físico para compensar los efectos del atracón. El tiempo medio de evolución de trastorno es de 26,7 meses $(D T=5,07)$ y más de la mitad de las pacientes tenía menos de 16 años cuando inician el trastorno, además de llevar más de dos años con el mismo. Además las puntuaciones respecto a la sintomatología asociada en el Revised Sympton Checklist-90 (SCL-90R) son bastante elevadas destacando principalmente las escalas de ansiedad, depresión y obsesivo-compulsivo. Los padres participantes fueron 27 , con una edad media de 45 años $(D T=5,05)$, siendo la mayoría mujeres $(69,57 \%)$; de éstas el 33,11\% es ama de casa. De los padres varones que conforman la muestra $(30,43 \%)$, la mayoría lo hacen acompañando a sus parejas, salvo dos de ellos $(7,4 \%)$ que, aunque acuden solos, comparten con las respectivas madres el cuidado de sus hijas. Todos los padres tienen estudios primarios completos y la mayoría de ellos $(81,48 \%)$ conviven con una pareja estable y sólo el 18,52\% están separados y/o divorciados o viven sin pareja. El 22,22\% de la muestra cuenta con un historial de antecedentes familiares con trastornos del comportamiento alimentario. Dos de las pacientes fueron excluidas de los análisis por encontrarse por debajo de los parámetros normales de peso y otras dos abandonan el grupo debido a la negativa de firmar el contrato de participación de sus padres en el estudio.

\section{Instrumentos de evaluación}

Tanto en la evaluación inicial como en el post-tratamiento y los seguimientos se utilizaron los mismos instrumentos, que se explican a continuación:

Para las pacientes:

Eating Disorder Examination (EDE; Fairburn y Cooper (1993). Esta entrevista semiestructurada permite obtener información cuantitativa de los aspectos claves del trastorno y evalúa de forma detallada la frecuencia e intensidad de las conductas específicas además de ajustarse a los criterios diagnósticos del DSMIV-TR. Consta de 62 preguntas divididas en 4 sub-escalas: restricción alimentaria, preocupación por el peso, preocupación por la comida y preocupación por la figura. Cuenta con buenos índices de fiabilidad $(\alpha=0,68-090)$ y validez concurrente $(r=0,38-0,82)$.

Bulimic Investigatory Test (BITE; Henderson y Freeman, 1987). Este cuestionario permite evaluar tanto los síntomas bulímicos (escala de síntomas) como la gravedad de los mismos (escala de gravedad). Está compuesta por 33 ítems y permite evaluar las conductas de sobreingesta y las características, tanto conductuales como cognitivas de la bulimia nerviosa. La sensibilidad al cambio terapéutico y su rápida aplicación, lo convierte en un cuestionario idóneo para monitorizar el tratamiento. Sus índices de fiabilidad para la escala de gravedad y de síntomas son de $\alpha=0,97$ y $\alpha=0,68$ respectivamente.

Revised Sympton Checklist-90 (SCL-90 R; Derogatis, 2001). A través de 90 ítems y ocho subescalas, evalúa la psicopatología general asociada al trastorno (somatización, obsesivocompulsivo, sensibilidad interpersonal, depresión, ansiedad, ansiedad fóbica, ideación paranoide y psicoticismo). Posee una consistencia interna de $\alpha=0,67$.

Autorregistro de Comida (Gómez, 2001). Permite evaluar las conductas alimentarias y 
obtener información objetiva para realizar el análisis funcional de las conductas bulímicas.

Para los padres:

Revised Sympton Checklist-90 (Derogatis, 2001).

Cuestionario sobre los Hábitos Alimentarios en la Familia: Elaborado ad hoc. Las conductas que se tuvieron en cuenta fueron las siguientes: Compra y elaboración de alimentos hipocalóricos, práctica de conductas restrictivas, ausencia de dietas equilibradas, exclusión de alimentos debido a su excesivo aporte calórico, grado de tensión durante las comidas, ingesta de cantidades superiores o inferiores a las cantidades normales, tiempo en realizar las comidas, número de comidas realizadas en familia.

Cuestionario de Percepción de Control del Problema Alimentario, elaborado ad hoc. El cuestionario evaluó la percepción subjetiva de los padres sobre: la gravedad del problema, la solución del mismo, respecto a si su actitud contribuye a la mejora del problema, sobre si posee información sobre el problema, sobre el impacto del trastorno en la relación con sus hijas.

Cuestionario de Observación de la Conducta Bulímica, elaborado ad hoc para evaluar la intensidad de los síntomas observados por los padres en su ambiente natural con respecto al: control de vómitos y atracones, comidas regulares, estado de ánimo y nivel de ansiedad.

\section{Procedimiento}

Se utilizó como grupo control a un grupo de adolescentes que habían recibido previamente la intervención protocolarizada. Este grupo siguió los mismos procedimientos de evaluación, criterios de inclusión, etc. que el grupo experimental. Asimismo, tenía las mismas características sociodemográficas y psicopatológicas que el grupo experimental. La diferencia entre ambos grupos radicaba en que el grupo control recibió la intervención unos meses antes y que sus padres no participaban en el estudio ni recibieron intervención psicoeducativa.

Para la selección de la muestra se contó con el equipo de profesionales de la Unidad de Trastornos Alimentarios del Hospital del Niño Jesús, que realizaron las primeras entrevistas diagnósticas. Aquellas pacientes que cumplían con los criterios diagnósticos de bulimia nerviosa del DSM-IV-TR fueron derivadas a las terapeutas para proceder entonces a la evaluación pre-tratamiento. Las pacientes con presencia de alto riesgo de suicidio, consumo de sustancias, que estuvieran siguiendo ya un tratamiento para la $\mathrm{BN}$ o cuyos padres no pudieran asistir al grupo de padres, fueron excluidas del estudio.

Las pacientes del grupo experimental fueron evaluadas y asignadas a los grupos en orden de llegada a la consulta del hospital, formando 3 grupos experimentales de 8,7 y 6 sujetos respectivamente.

El proceso de evaluación se llevó a cabo mediante la entrevista semi-estructurada a nivel individual y la aplicación de los autoinformes y autorregistros a nivel grupal. En el caso de exclusión se remitía a las pacientes nuevamente al equipo de primeras entrevistas para su reasignación a otra modalidad de tratamiento. El tratamiento cognitivo-conductual para las pacientes fue elaborado por Gómez (2001) a partir del programa de autoayuda de Fairburn (1995). La intervención con los padres fue desarrollada a partir de una guía para padres (Gómez, Castro, Garcia, Dúo y Yela, 2003).

Las sesiones para las pacientes se dividen en tres fases siguiendo el modelo de Fairburn. La primera tiene que ver con el control de la sintomatología alimentaria: se hace hincapié en la información sobre la enfermedad y sus consecuencias y las ventajas de introducir un patrón regular de comidas, planificación de actividades incompatibles con el atracón como estrategia de prevención de respuesta, entrenamiento en habilidades y estrategias eficaces para la solución de problemas y se inicia el abandono gradual tanto de las conductas restrictivas como las conductas de evitación al cuerpo, peso y/o comida (sesiones 1 a 6). En la segunda fase, el objetivo es la reestructuración cognitiva de las ideas irracionales que funcionan como mantenedoras del problema (Perpiñá, Roncero y Belloch, 2008) y que tienen que ver con la insatisfacción corporal y la alimentación (sesiones 7 a 11) y en la última fase, se aborda la prevención de recaídas (sesión 12).

En el caso del grupo que recibió tratamiento paralelo de padres, estas sesiones se realiza- 
ban inmediatamente después de las sesiones con las pacientes por la misma terapeuta que trataba al grupo experimental. En un primer momento, se daba información general, tanto sobre la evolución como sobre los contenidos trabajados en cada sesión con las hijas (10-15 min.). En segundo lugar, se revisaban las dudas o dificultades con relación a las estrategias de entrenamiento de la semana anterior (10-15 min.). En tercer lugar, se distribuía el material correspondiente a la sesión de ese día; se explicaban los objetivos de cada una de las estrategias de control recomendadas y se entrenaba su puesta en marcha (45-50 min.). Finalmente se hacía un breve resumen, reservando unos minutos para resolver dudas o dificultades.

El contenido de las sesiones para padres se divide también en 3 fases. La primera (sesiones 1 a 6) tiene que ver con información general de la enfermedad y sus consecuencias, haciendo hincapié sobre las conductas familiares mantenedoras del problema (refuerzo de la delgadez y las dietas restrictivas, horarios irregulares, pocas comidas en familia, falta de control y de orden en las comidas). La segunda fase (sesiones 7 a 11) tiene como objetivo el aprendizaje de estrategias que permitan crear un ambiente familiar que facilite el cambio y que les permita ejercer como modelos adecuados a la hora de las comidas. En las sesiones de esta fase, el trabajo está encaminado a que los padres puedan sustituir los hábitos inadecuados por hábitos saludables a través del aprendizaje de estrategias que permitan reducir la ansiedad durante las comidas y un entrenamiento para manejar de manera más coherente y eficaz las conductas bulímicas. La tercera (sesión 12) aborda la prevención de recaídas. En la Tabla 1 se resume el contenido de cada una de las sesiones.

Ambos programas constaron de 12 sesiones de 90 minutos con una periodicidad semanal. Las intervenciones tuvieron carácter grupal con un mínimo y máximo de 6-10 sujetos por grupo respectivamente.

En las sesiones con las pacientes siempre se procedía de la misma manera: se pesaba a las pacientes como estrategia de exposición al peso, se revisaban los autorregistros, y se evaluaba la frecuencia de vómitos y atracones semanales (15-20 min.). Se comunicaba a las pacientes su peso para que comprobaran que este no variaría de manera significativa como resultado de la aplicación de las pautas del tratamiento. Luego se revisaban las dificultades semanales en relación a la puesta en marcha de las técnicas de afrontamiento de la sesión anterior (10-15 min.). En tercer lugar se explicaban los objetivos de la sesión actual, se procedía al entrenamiento de las técnicas comentadas y a la entrega de material (40-45 min.). Se finalizaba con un re-

Tabla 1. Resumen del contenido de las sesiones para los padres y el paciente

\begin{tabular}{|c|c|c|}
\hline Sesión & Pacientes & Padres \\
\hline 1 & Registro de comidas & Motivación \\
\hline 2 & Introducción patrón regular comidas & Información general de la enfermedad (I) \\
\hline 3 & Planificación actividades incompatibles con el atracón & Información general de la enfermedad (II) \\
\hline 4 & Solución de problemas & Circulo vicioso que mantiene el atracón \\
\hline 5 & Abandono de la dieta y conductas de evitación & $\begin{array}{l}\text { Identificación de hábitos familiares mantenedores del } \\
\text { problema (I) }\end{array}$ \\
\hline 6 & Identificación de emociones negativas & $\begin{array}{l}\text { Identificación de hábitos familiares mantenedores del } \\
\text { problema (II) }\end{array}$ \\
\hline 7 & Identificación de pensamientos automáticos & Aprendizaje de hábitos saludables \\
\hline 8 & Distorsiones cognitivas (I) & Estrategias para comer despacio \\
\hline 9 & Distorsiones cognitivas (II) & Estrategias para el manejo de conductas bulímicas \\
\hline 10 & Desmontando distorsiones cognitivas (I) & Identificación y manejo del pensamiento obsesivo (I) \\
\hline 11 & Desmontando distorsiones cognitivas (II) & Identificación y manejo del pensamiento obsesivo (II) \\
\hline 12 & Prevención de recaídas & Prevención de recaídas \\
\hline
\end{tabular}


sumen de la sesión y se dejaban los últimos minutos para resolver dudas (10-15 min.). La terapeuta llevaba un control semanal tanto del peso, como de la frecuencia de vómitos y atracones semanales.

Al concluir la intervención se realizó la evaluación post-tratamiento, tanto con las pacientes como con sus respectivos padres, y se acordaron las fechas de la evaluación de seguimiento (3, 6 y 12 meses). Los instrumentos de evaluación fueron los mismos que la evaluación inicial.

\section{Resultados}

Previo al análisis de los datos, se procedió al estudio de la homogeneidad de los grupos mediante la prueba $t$ para muestras independientes para las variables cuantitativas, y la prueba Chi-cuadrado $\left(\chi^{2}\right)$ para las variables cualitativas, no encontrándose diferencias estadísticamente significativas en ninguna de las variables analizadas, lo que indica que los grupos son homogéneos. Asimismo se analizó si las muestras se ajustaban a una distribución normal. Para ello se utilizó la prueba de Kolmogorov-Smirnov como prueba de bondad de ajuste, pudiéndose asumir la distribución normal de las muestras en las variables cuantitativas del pretratamiento $(\mathrm{p}<0,01)$.

Para el análisis estadístico sobre la eficacia del tratamiento se utilizaron las siguientes pruebas: (a) $t$ de Student para muestras relacionadas, para evaluar los cambios tras el tratamiento en los períodos de seguimiento y el tamaño del efecto con el estadístico G*POWER (Faul y Erdefeldef, 1992). (b) $t$ de Student para muestras independientes para evaluar la eficacia añadida del grupo de padres (TCC+GP vs TCC). (c) Chi-cuadrado $\left(\chi^{2}\right)$ para el análisis de recaídas y abandonos.

Con respecto al grupo experimental aparecen cambios significativos, entre línea base y postratamiento, en todas las variables evaluadas, tanto en aquellas referidas a los síntomas centrales de la bulimia como a las relacionadas con síntomas asociados (tabla $n^{\circ} 2$ ). Se realizó una prueba de $t$ para estudiar la evolución de los vómitos y los atracones y se comprobó que estas diferencias se mantienen en los seguimientos de los 3, 6 y 12 meses. (Tabla 3)

Si comparamos los resultados entre los grupos (TCC+GP vs TCC), no se observan diferencias estadísticamente significativas en los

Tabla 2. Medias y desviaciones típicas (entre paréntesis) pre-postratamiento para el grupo experimental $(n=21)$, prueba de $t$ y tamaño del efecto

\begin{tabular}{lllll}
\hline Variable & Pre-tratamiento & Post-tratamiento & $t(20)$ & Tamaño del efecto $(d)$ \\
\hline Vómitos/ semana & $5,26(5,07)$ & $0,79(1,50)$ & $11,99^{* *}$ & 0,88 \\
Atracones/ semana & $5,27(2,7)$ & $0,88(1,56)$ & $6,69 * *$ & 1,62 \\
BITE & & & \\
Escala de síntomas & $21,14(5,61)$ & $12,19(6,86)$ & $5,46^{* *}$ & 1,59 \\
Escala de gravedad & $10,86(5,02)$ & $3,71(2,83)$ & $7,02 * *$ & 1,27 \\
Puntuación total & $32,00(7,02)$ & $15,90(8,28)$ & $7,47^{* *}$ & \\
EDE & & & & 1,07 \\
Preocupación comida & $3,88(1,39)$ & $2,39(1,54)$ & $4,78^{* *}$ & 1,25 \\
Preocupación peso & $4,12(1,31)$ & $2,48(1,48)$ & $5,34^{* *}$ & 1,49 \\
Preocupación figura & $4,57(1,20)$ & $2,78(1,38)$ & $6,68^{* *}$ & $1,99 * *$ \\
Restricción alimentaria & $4,25(1,46)$ & $1,09(1,05)$ & & 1,60 \\
SCL-90R & & & $3,61 * *$ & \\
Escala global de gravedad (GSI) & $1,60(0,80)$ & $1,06(0,53)$ & & \\
\hline
\end{tabular}

Nota. Tamaño del efecto: grande $>0,8$, mediano $\geqslant 0,5$, pequeño $<0,5$.

$* * p<0,01$. 
Tabla 3. Diferencias en las puntuaciones medias para el grupo experimental en función de los momentos temporales de medida

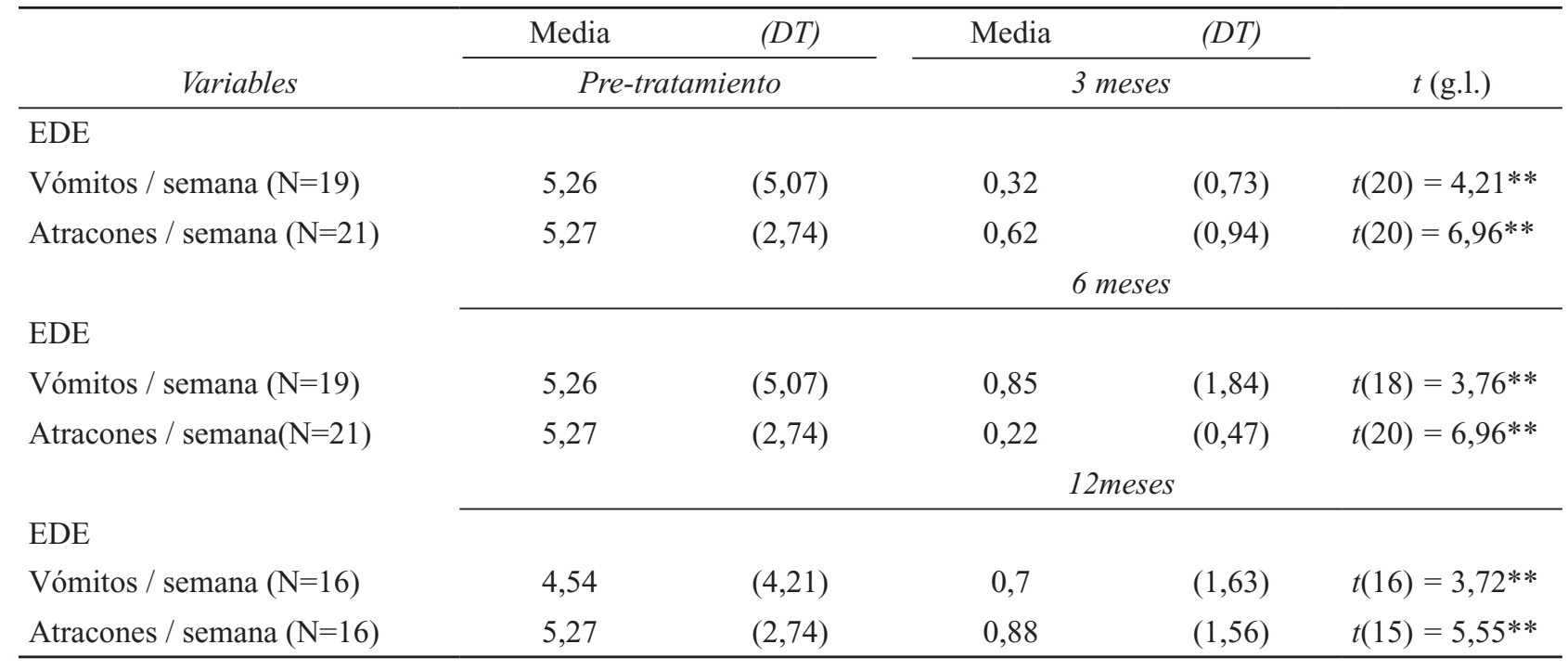

$* * \mathrm{p}<0,01$.

síntomas centrales del trastorno (vómitos y atracones), síntomas bulímicos y psicopatología asociada (Tabla 4).

Sin embargo, se observan diferencias con respecto a los abandonos en todos los momentos de seguimiento, acentuándose con el paso del tiempo, alcanzando la significación estadística a partir del seguimiento de los 6 meses (Tabla 4). En el análisis de recaídas (reducción de vómitos/atracones inferior al 50\%, Blouin, 1994), también se pueden observar diferencias entre los grupos en todos los momentos del seguimiento, en los que el porcentaje de recaídas del grupo TCC $+\mathrm{GP}\left(N_{T C C+G P}=21\right)$ es siempre inferior que en el grupo TCC $\left(N_{T C C}=17\right)$. Con el paso del tiempo las diferencias son cada vez más importantes, alcanzado la significación estadística a partir de 6 meses de seguimiento en el caso de los atracones y a partir de los 12 meses de seguimiento en el caso de los vómitos.

Respecto a los resultados obtenidos por los padres, tras el tratamiento se produce un cambio estadísticamente significativo en la percepción de control de los padres sobre el problema de sus hijas, se incrementa el nivel de información que poseen los padres, y se experimenta una mejora en las estrategias de afrontamiento del problema; además, se aprecia una disminución significativa en el impacto del trastorno alimentario en las relaciones entre padres e hijos.

Los padres corroboran los cambios que experimentan las conductas de las pacientes, ya que, según los datos del cuestionario sobre observación de la conducta bulímica, se aprecian diferencias estadísticamente significativas en todas las variables que tienen relación directa con los síntomas alimentarios (control vómito y atracón, conducta alimentaria), y el grado de ansiedad y depresión que los padres observan en sus hijas (Tabla 5).

Esta tabla además resume los cambios más importantes que tienen que ver con los hábitos alimentarios en la familia, dentro de los cuales destaca, por un lado, el incremento de las comidas realizadas en familia y una mejora de la dieta hacia una alimentación más equilibrada y, por otro, la disminución del número de alimentos "prohibidos" a los que, por considerarse hipercalóricos, se les asocia un efecto negativo sobre la figura y el cuerpo.

\section{DISCUSIÓN}

\section{Eficacia de la intervención}

El programa de tratamiento de la presente investigación, fue elaborado a partir del progra- 
Tabla 4. Diferencias en las puntuaciones medias durante el postratamiento entre los grupos experimental y control (Grupo TCC + GP vs. TCC) $(n=38)$

\begin{tabular}{|c|c|c|c|}
\hline & Grupo TCC+GP & Grupo TCC & \\
\hline Variable & Media $(D T)$ & Media $(D T)$ & $t(36)$ \\
\hline \multicolumn{4}{|l|}{ BITE } \\
\hline Síntomas & $12,19(6,86)$ & $16,41(7,45)$ & $-1,81$ \\
\hline Gravedad & $3,71(2,83)$ & $4,94(2,92)$ & $-1,30$ \\
\hline Puntuación total & $15,90(8,28)$ & $21,35(9,49)$ & $-1,90$ \\
\hline \multicolumn{4}{|l|}{ EDE } \\
\hline Vómitos/semana & $0,79(1,50)$ & $0,75(1,10)$ & $-0,10$ \\
\hline Atracones/semana & $0,88(1,56)$ & $0,92(1,16)$ & 0,28 \\
\hline Preocupación por la comida & $2,39(1,54)$ & $1,58(1,58)$ & 1,57 \\
\hline Preocupación por el peso & $2,48(1,48)$ & $3,01(1,73)$ & $-1,01$ \\
\hline Preocupación por la figura & $2,78(1,54)$ & $3,35(1,70)$ & $-1,12$ \\
\hline Restricción alimentaria & $1,09(1,05)$ & $1,27(1,06)$ & $-1,51$ \\
\hline \multicolumn{4}{|l|}{ SCL-90 } \\
\hline \multirow[t]{2}{*}{ Escala global de gravedad } & $1,06(0,53)$ & $1,18(0,66)$ & $-6,16$ \\
\hline & Media $(D T)$ & Media $(D T)$ & $\chi^{2}(1)$ \\
\hline \multicolumn{4}{|l|}{ Recaídas atracones } \\
\hline Seguimiento 3 meses & $9,50 \%$ & $33,30 \%$ & 3,75 \\
\hline Seguimiento 6 meses & $14,30 \%$ & $50 \%$ & $5,51 *$ \\
\hline Seguimiento 12 meses & $14,30 \%$ & $50 \%$ & $5,51^{*}$ \\
\hline \multicolumn{4}{|l|}{ Recaidas vómitos } \\
\hline Seguimiento 3 meses & $10,59 \%$ & $25 \%$ & 1,2 \\
\hline Seguimiento 6 meses & $15,80 \%$ & $37,50 \%$ & 2,06 \\
\hline Seguimiento 12 meses & $21,05 \%$ & $56,25 \%$ & $6,52 *$ \\
\hline \multicolumn{4}{|l|}{ Abandonos } \\
\hline Seguimiento 3 meses & $5 \%$ & $22 \%$ & 3,2 \\
\hline Seguimiento 6 meses. & $10 \%$ & $39 \%$ & $5,79 *$ \\
\hline Seguimiento 12 meses & $14 \%$ & $44 \%$ & $5,37^{*}$ \\
\hline
\end{tabular}

$* p<005$

ma de Fairburn (1995), adaptándolo específicamente para la población adolescente, incluyendo dos novedades importantes: el formato grupal breve y la inclusión de un grupo paralelo de padres.

La intervención en modalidad grupal, mejora su eficiencia, sobre todo si consideramos que el $92 \%$ de los estudios controlados derivados del trabajo de Fairburn son llevados a cabo de manera individual, lo que supone un tratamiento más costoso que el propuesto en la presente in- vestigación. En este sentido, Chen et al. (2003) calculan un ahorro de entre un $70-80 \%$ si se compara la terapia grupal con la terapia individual, por lo que, en nuestra opinión, el formato grupal debería ser considerado como la primera elección antes de decantarse por formatos alternativos. Además, la brevedad de la intervención (24 sesiones: 12 sesiones para las pacientes y 12 sesiones para sus padres, en 3 meses) permite que los sujetos se puedan reincorporar exitosamente a sus actividades habituales y afrontar una 
Tabla 5. Diferencias en las puntuaciones medias en el grupo de padres para las diferentes variables (prueba de $t$ )

\begin{tabular}{|c|c|c|c|}
\hline Variable & $\begin{array}{c}\text { Pre-tratamiento } \\
\text { Media (DT) }\end{array}$ & $\begin{array}{c}\text { Post-tratamiento } \\
\text { Media (DT) }\end{array}$ & $t(20)$ \\
\hline \multicolumn{4}{|c|}{ Cuestionario de Percepción de Control del Problema Alimentario (0-10) } \\
\hline Percepción sobre el gravedad del trastorno & $6,95(1,85)$ & $5,71(2,28)$ & $3,20 * *$ \\
\hline Respecto a la solución del problema & $8,04(1,77)$ & $8,42(1,50)$ & 4,23 \\
\hline Respecto a si su actitud contribuye & $6,00(2,21)$ & $8,23(1,86)$ & $-3,40 * *$ \\
\hline Respecto a si posee información sobre el problema & $5,19(2,35)$ & $9,09(1,09)$ & $-6,33 * *$ \\
\hline Respecto su eficacia en relación al manejo del problema & $4,95(2,95)$ & $7,66(1,19)$ & $-4,05 * *$ \\
\hline Impacto negativo sobre la relación con ella & $6,28(2,36)$ & $4,71(2,84)$ & $2,17 *$ \\
\hline \multicolumn{4}{|c|}{ Cuestionario sobre Hábitos Alimentarios en la Familia (0-10) } \\
\hline Comidas en familia & $4,50(2,41)$ & $7,11(1,85)$ & $-4,79 * *$ \\
\hline Grado de tensión en las comidas & $5,09(3,74)$ & $3,13(2,86)$ & $3,04 * *$ \\
\hline Dieta equilibrada & $7,59(2,55)$ & $9,27(1,12)$ & $-2,83 * *$ \\
\hline Compra de alimentos hipocalóricos & $7,40(3,18)$ & $2,50(3,20)$ & $6,59 * *$ \\
\hline Modificación de hábitos alimentarios & $3,63(3,43)$ & $1,72(2,86)$ & $2,41 *$ \\
\hline Cantidades comidas & $5,45(1,14)$ & $5,63(0,95)$ & 0,00 \\
\hline \multicolumn{4}{|c|}{ Cuestionario de Observación de la Conducta Bulímica (0-7) } \\
\hline Control de atracones & $2,58(1,41)$ & $5,29(1,36)$ & $-7,25^{* *}$ \\
\hline Control de vómitos & $3,29(1,33)$ & $5,79(1,25)$ & $-6,33 * *$ \\
\hline Comidas regulares & $3,43(1,64)$ & $5,86(1,32)$ & $7,71 * *$ \\
\hline Mejora del problema & $3,66(1,16)$ & $5,70(1,23)$ & $-5,26 * *$ \\
\hline Nivel de ansiedad & $4,95(1,36)$ & $4,12(1,59)$ & $2,17 *$ \\
\hline Estado de ánimo global & $4,41(1,50)$ & $3,54(1,85)$ & $2,38^{*}$ \\
\hline
\end{tabular}

$* \mathrm{p}<0,05 ; * *_{\mathrm{p}}<0,01$.

vida social, que ha estado especialmente deteriorada, lo que tiene una significación muy importante durante la adolescencia.

Esta brevedad es aún más importante si se considera que, en la práctica clínica habitual, el número de sesiones, con las pacientes, suele ser mayor que el propuesto en este estudio (alrededor de 40 sesiones, con una duración cercana al año). Por ello, un tratamiento como el aquí propuesto supone, claramente, un ahorro de tiempo y sufrimiento para las pacientes. Además, el trabajo con menores de edad requiere algún tipo de intervención con sus progenitores, que en la práctica clínica suele realizarse de manera individual y no estructurada, con un tiempo promedio mucho más largo y, por tanto más costoso, que el aquí propuesto.

La intervención se ha mostrado altamente eficaz ya que los resultados indican que, tras el tratamiento, disminuyen de manera significativa tanto los vómitos como los atracones, que se reducen en un $83,3 \%$ y $84,9 \%$ respectivamente, tasas ligeramente superiores a las encontradas en la revisión bibliográfica que oscilan entre un $13-70 \%$ para los vómitos y un 20 y un $70 \%$ para los atracones, con una media de sesiones superior a 16 (Lundgren, Dannoff y Drew, 2004).

Es llamativa la importante reducción de los síntomas bulímicos, casi desde la segunda sesión de tratamiento, y confirmaría las hipótesis de los estudios que destacan el cambio en las primeras sesiones como uno de los mejores predictores de la respuesta al tratamiento (Fairburn, Agras, Walsh, Wilson y Stice, 2004). Por tanto, para conseguir mejores resultados, se tendría que llevar a cabo un esfuerzo especial en maximizar el cambio en las primeras sesiones, además de prestar ayuda adicional y com- 
plementaria a las pacientes que no respondan de manera adecuada durante este período.

También se consigue una reducción significativa de la psicopatología asociada. Este resultado es interesante, si se considera que el tratamiento únicamente aborda los aspectos relacionados con el trastorno. Se corroboran así las hipótesis que consideran la psicopatología como secundaria al trastorno, ya que parece poco probable que estos síntomas comórbidos hubiesen disminuido sin el cese o reducción de la frecuencia de las conductas bulímicas.

Un aspecto importante a destacar es que la intervención con los padres produce cambios importantes en algunos de los hábitos alimentarios y de las reacciones familiares que han favorecen el mantenimiento y el refuerzo del trastorno (Cervera, 2005). En tal sentido se consigue por un lado, incrementar el número de comidas realizadas en familia, disminuir el grado de tensión durante las mismas, introducir una dieta familiar más equilibrada y variada y por otro una mejora en todas las variables relacionadas a la percepción de control de los padres sobre el problema de sus hijas (ver Tabla 4). Estas situaciones permiten crear un ambiente mucho más favorable al cambio, ya que permiten que la paciente tenga más posibilidades de introducir los "alimentos prohibidos" en su dieta bajo una situación de mayor control, lo que favorece tanto la disminución de los niveles subjetivos de ansiedad como el reaprendizaje de las sensaciones de hambre y saciedad.

Uno de los logros más importantes del tratamiento tiene que ver con la adherencia al mismo. La gran mayoría de las pacientes $(90,5 \%, n=19)$ asistió a más del $80 \%$ de las sesiones. Además, el porcentaje de abandonos $(8,69 \%, n=2)$ es especialmente bajo en comparación con los estudios revisados, que oscilan entre un $27 \%$ para las terapias grupales (Chen et al., 2003) y un 33\% para las terapias individuales (Whittal, Agras Gould., 1999). Si tenemos en cuenta que una de las pacientes abandona antes de empezar el tratamiento, el porcentaje de abandonos durante el mismo se reduce al $4,54 \%(n=1)$. Estas diferencias podrían tener que ver con el hecho de que los datos de la literatura, como ya se mencionó, hacen referencia a muestras adultas, pero tam- bién pueden deberse a la mejoría que las pacientes experimentan en las primeras sesiones.

\section{Eficacia diferencial del programa de padres}

Si se comparan la eficacia de las dos modalidades de intervención (TCC+GP vs TC), no se observan diferencias estadísticamente significativas en ninguna de las variables evaluadas con respecto a los síntomas y psicopatología asociada. Sin embargo se encuentran diferencias importantes en cuanto al porcentaje de recaídas en todos los momentos a favor del TCC + GP, acentuándose las diferencias con el paso del tiempo.

Si se analizan las diferencias con relación al porcentaje de abandonos entre ambos grupos, también podemos apreciar que éstas son importantes en todos los momentos del seguimiento, siendo siempre superior el porcentaje de abandonos del grupo TCC que el TCC+GP. Al igual que en el caso de las recaídas, las diferencias se incrementan gradualmente con el tiempo, encontrándose diferencias estadísticamente significativas a partir de los 6 meses de seguimiento. Una posible explicación a la mayor eficacia del grupo de padres es el hecho de que el trabajo con padres supone un control externo adicional a las terapias cognitivo conductuales convencionales, lo que dificulta a la paciente la realización de conductas bulímicas.

Por tanto, se puede afirmar que el tratamiento con padres, no solamente es eficaz en sus resultados, sino que disminuye el porcentaje tanto de abandonos como de recaídas. Si bien es cierto que es un tratamiento más largo que la intervención cognitivo-conductual en solitario ( $\mathrm{TCC}=12$ sesiones vs $\mathrm{TCC}+\mathrm{GP}=24$ sesiones), no lo es menos que su aplicación permite dar respuesta adecuada a una de las demandas más importantes de la comunidad científica: plantear tratamientos que mantengan sus beneficios con el paso del tiempo.

Otro aspecto relevante del trabajo con los padres tiene que ver con instaurar una serie de normas con respecto a los hábitos alimentarios, para así romper la asociación que las pacientes suelen hacer con la comida y las claves contextuales del atracón. Ello implica comidas regu- 
lares, en un mismo lugar de la casa, y que la paciente se siente a la mesa frente al plato, independientemente de que decida comer o no, hasta que pase el tiempo necesario para levantarse de la mesa. De este modo, durante todo este tiempo estará exponiéndose al alimento. Además, se dan instrucciones a los padres sobre cómo han de llevar a cabo la supervisión de las comidas y cómo han de afrontar situaciones difíciles que puedan surgir en ellas.

Estas medidas, en definitiva, disminuyen las conductas de evitación de la paciente, y facilitan la exposición gradual y constante, no sólo a la comida, sino también al peso y figura. Se puede concluir, por tanto, que esta intervención estructurada, dirigida tanto a las pacientes como los padres, potencia los resultados terapéuticos en relación al tratamiento estándar y favorece el mantenimiento de los mismos en el seguimiento.

Las limitaciones de la intervención son varias e importantes, entre ellas se pueden señalar: (1) El tamaño de la muestra, ya que una muestra más grande podría haber incrementado la potencia estadística, y, resaltar las diferencias entre ambas modalidades de tratamiento; (2) La falta de un grupo control de padres, puesto que el grupo control se obtuvo de una investigación previa; (3) La falta de validación de los cuestionarios para padres no permite que se puedan extraer de ellos resultados concluyentes; Y (4) el hecho de que la intervención se llevara a cabo por distintas terapeutas (en el grupo experimental y el grupo control) podría explicar algunas de las diferencias encontradas entre ambos grupos.

A pesar de estas limitaciones, y a la vista de los resultados obtenidos, parece que las intervenciones propuestas (y especialmente la que incluye grupo de padres) son altamente eficaces y eficientes en el tratamiento de la bulimia nerviosa. Sería conveniente la realización de un estudio, que salvando las limitaciones expuestas, permitiera confirmar los resultados aquí encontrados.

\section{REFERENCIAS}

American Psychiatric Association, APA (2000a). Practice guideline for the treatment of patients with eating disorders. American Journal of Psychiatry, 157. Suplemento
American Psychiatric Association, APA (2000b). Diagnostic and statistical manual of mental disorders: ( $4^{\text {th }}$ Ed.). Washington, DC: APA

Bryant-Waugh, R. (2006). Eating disorders in children and adolescents. En S. Wonderlich, J. E. Mitchell, M. de Swaam y H. Steiger (Eds). Annual Review of Eating Disorders: Part 2. (pp. 96-112).

Calvo, R. (2002). Anorexia y bulimia: Guía para padres, educadores y terapeutas. Madrid: Planeta.

Cervera, M. (2005). Riesgo y prevención de la anorexia y la bulimia. Madrid: Pirámide

Chen, E, Touyz, S.W, Beumont, P.J, Fairburn, C.G, Griffiths, R, Butow, P, Russel, J, Schoote, D.E, Gertler, R, Basten, C. (2003). Comparison of group and individual cognitivebehavioral therapy for patients with bulimia nervosa. International Journal of Eating Disorders, 33, 241-254.

Colahan, M.A., Robinson, P. H. (2002). Multi-family groups in the treatment of young adults with Ealing disorders. Journal of Family Therapy, 24, 17-30.

Derogatis, L.R. (2001). Cuestionario de 90 síntomas (SCL-90-R). Madrid: TEA Ediciones

Eisler, I., Le Grange, D y Asen, E. (2003). Family interventions. En: J. Treasure, U. Smith y E van Furth (Eds.), Handbook of eating disorders ( $\left.2^{\mathrm{a}} \mathrm{Ed}\right)$. Chichester: John Wiley and Sons

Fairburn, C.G. (1981). A cognitive-behavioral approach to the management of bulimia. Psychological Medicine, 11, 707-711.

Fairburn, C.G. y Cooper, Z. (1987). Behavioral and cognitive approaches to the treatment of anorexia nervosa and bulimia nervosa. En P. J. V. Beamont, G. D. Burrows y R. C. Casper (Eds.), Handbook of eating disorders. Amsterdam: Elsevier.

Fariburn, C.G y Cooper, Z (1993). The Eating Disorder Examination (12 ${ }^{\text {th }}$ Ed.). En Fairburn, C.G y Wilson (Eds).Binge Eating: Nature, assessment and treatment. (pag 317-360). Nueva York: Guiford Press.

Fairburn, C.G. (1995). Overcoming binge eating. New York: Guilford Press.

Fairburn, C.G., Agras, W., Walsh, T., Wilson, G.T. y Stice, E. (2004). Predictor of outcome in bulimia nervosa by early change in treatment. American Journal of Psychiatry, 161, 2322-2324.

Faul, F. y Erdefelder, E. (1992). G*POWER: A priori, post-hoc and compromise power for MS-DOS. Bonn: Bonn University.

Gómez, M (2001). Eficacia del tratamiento cognitivo-conductual en un grupo de pacientes con bulimia nerviosa. Tesis Doctoral. Universidad Pontificia de Salamanca

Gómez, M.A., Castro, U., García, A., Dúo, I. y Yela, J.R. (2003). ¿Qué es la bulimia? Un problema con solución. Madrid: Pirámide.

Henderson, M. y Freeman, C.P.L. (1987). A self-rating scale for bulimia: the BITE. British Journal of Psychiatry, 150, 18-24. 
Iglesias, B y Romero, E. (2009). Estilos parentales percibidos, psicopatología y personalidad en la adolescencia. Revista de Psicopatología y Psicología Clínica, 14, 63-77.

Le Grange, D (2005). Family issues and eating disorders. En S. Wonderlich, J. Mitchel, de Zwann y H. Steiger (Eds). Eating Disorders Review: Part I. Oxford: Radcliffe Publishing.

Lock, J y Le Grange, D. (2005). Help your teenager bet an ED. Nueva York: Guilford Press.

Lundgren, J.D., Danoff-Burg, S y Drew, A. (2004). Cognitive-behavioral therapy for bulimia nervosa: An empirical analysis of clinical significance. International Journal of Eating Disorders, 35, 262- 274.

NICE (National Institute for Clinical Excellence) (2004). Eating Disorders: core interventions in the treatment and management of anorexia nervosa, bulimia nervosa and related eating disorders. London: British Psychological Society.

Okon, D., Greene, A. y Smith, J.E. (2003). Family interactions predict intraindividual symptom variation for adolescents with bulimia. International Journal of Eating Disorders, 34, 450-457.

Perpiñá, C., Roncero, M. y Belloch, A. (2008) Intrusiones sobre trastornos alimentarios en población general: desarrollo y validación del Inventario de Pensamientos Intrusos Alimentarios (INPIAS). Revista de Psicopatología y Psicología Clínica, 13, 187-203.

Rusell, G.F., Szmukler, G.I., Dare, C., Eisler, I. (1987). An evaluation of family therapy in anorexia nervosa and bulimia nervosa. Archives of General Psychiatry, 44, 1047-1056.

Shapiro, J.R., Berkman, N.D., Brownley,K.A., Sedway, J.K., Lohr, K.N., Bulik,C.M. (2007). Bulimia Nervosa Treatment: A Systematic Review of Randomized Controlled Trials. International Journal of Eating Disorders; 40, 321-336.

Toro, J. (1996). El cuerpo como delito. Barcelona: Ariel.

Vadereycken, W., Castro, J. y Vanderlinden (1991). Anorexia y bulimia. Barcelona: Martínez Roca.

Vanderlinden, J y Vandereycken, W (1999). Trauma, disociación y descontrol de Impulsos en los Trastornos Alimentarios. Nuevas terapias para el control de la anorexia y de la bulimia. Barcelona: Ediciones Granica, S.A.

Whittal, M.L., Agras, W.S y Gould, R.A. (1999). Bulimia nervosa: A meta-analysis of psychosocial and pharmacological treatments. Behavior Therapy, 30, 117-136.

Wilson, G.T. y Fariburn, C.G. (1998). Treatment of eating disorders. En P.E. Nathan y J.M. Gorman (Eds). A guide to treatments that work (pag. 501-530). Nueva York: Oxford University Press.

Wilson, G.T. (2005). Psychological treatment of eating disorders. Annual Review of Clinical Psychology, 1, 439-465.

Winn, S., Perkins, S., Schmidt, U., Eisler, F., Treassure, J, Berelowitz, M. (2007). Predictors of mental health problems and negative caregiving experiences in carers of adolescents with bulimia nervosa. Interntational Jouranl of Eating Disorders, 40, 171-178. 